\title{
Health Care Providers' Understanding of Health Care Beliefs and Preferences in Anabaptist Communities
}

\author{
Dawn Garrett-Wright, PhD, PMHNP-BC, CNE \\ Professor \\ Western Kentucky University School of Nursing and Allied Health \\ M. Eve Main, DNP, APRN-BC \\ Professor \\ Western Kentucky University School of Nursing and Allied Health \\ M. Susan Jones, PhD, RN, CNE, ANEF \\ Professor Emerita \\ Western Kentucky University School of Nursing and Allied Health \\ Corresponding Author \\ Dawn Garrett-Wright \\ dawn.garrett@wku.edu \\ WKU SONAH \\ 1906 College Heights Blvd \\ Bowling Green, KY 42101 \\ 270-745-3800
}

\begin{abstract}
Anabaptist communities are among the fastest growing communities in the United States. Reviews of medical and sociological literature consistently demonstrate that members of Anabaptist communities hold health care beliefs and preferences that differ from the mainstream culture. However, the professional health care literature is lacking in information regarding health care providers' understanding of health care beliefs and preferences in Anabaptist groups. The purpose of this study was to explore the understanding of Anabaptist patients' health care beliefs and preferences as well as lessons learned from providers who have experience delivering health care for this culturally diverse population. A qualitative descriptive approach was used to collect data with a sample of health care providers $(N=18)$ selected via purposive sampling. The study participants provided informed consent to be interviewed and videotaped responding to a set of semi-structured interview questions. The videotaped interviews were transcribed before thematic analysis was conducted independently and then collectively by a group of three researchers familiar with Anabaptist health care practices. Through thematic analysis, seven themes were identified among the participants relating to their understanding of the beliefs and preferences of Anabaptist community members they served.
\end{abstract}

Submitted February 24, 2020; accepted June 3, 2020; published September 18, 2020 https://doi.org/10.18061/jpac.v1i1.7537

Keywords: Anabaptist, health care providers, health care beliefs and preferences, perceptions 


\section{Introduction}

Culture provides a form of meta-communication within a group and includes aspects such as the customs, attitudes, and beliefs of group members (Spector, 2017). Health practices and interactions with health care providers (HCPs) are filtered through the lens of culture. The ability to provide culturally competent and sensitive health care is an expected standard of care for HCPs (Edwards, 2013; Spector, 2017; Unver et al., 2019). Delivery of culturally competent health care entails both an understanding of and giving attention to the total patient context (Spector, 2017). Culturally sensitive health care requires the HCP to have "basic knowledge of and constructive attitudes toward the health traditions" of the population receiving service (Spector, 2017, p. 267). Lack of cultural competence and sensitivity on the part of HCPs has been reported to lead to negative consequences for patients and the health care system (Flynn et al., 2019), while culturally informed care has been noted to improve patient outcomes and satisfaction (Rohr et al., 2019). To respond to the increasing need for culturally competent and sensitive care, HCPs must seek to create a "patient-centered friendly" environment and to minimize their own biases regarding diverse cultural groups (Edwards, 2013, p. 38).

Anabaptist communities, composed largely of Amish and Mennonite groups, represent a small but flourishing segment of the United States (U.S.) population (Lancaster Mennonite Historical Society, 2019). This population has been noted to have unique perceptions and preferences related to health care when compared to the mainstream U.S. population (Donnermeyer \& Friedrich, 2006; Garrett-Wright et al., 2016). However, the literature is limited related to HCPs' understanding of the underlying beliefs and preferences related to health care of Anabaptists. In addition, it is noted that many HCPs may have limited experience in working closely with Anabaptist communities. The purpose of this two-fold qualitative study was to explore the various perceptions of health care beliefs and preferences for Anabaptist community members from both the perspective of the community members and that of the HCP. The results of the written questionnaire that explored Anabaptist community members' experiences with and preferences related to health care services have been previously reported (Garrett-Wright et al., 2016). The current article focuses on the analysis of interviews with HCPs who were experienced in caring for Anabaptist groups and their understanding as providers of what their Anabaptist patients valued and desired in health care interactions.

\section{Background}

Consistency has been found among reported health care beliefs and preferences of Anabaptists. A review of literature indicates that a key component of the health care philosophy of an Anabaptist community member includes responsibility for one's own health along with a respect for the role that God's will plays in one's life and health status (Fisher, 2002; Sherman, 2014). Anabaptists live by a basic principle of separation of church and state. These core beliefs deter most Anabaptist community members from accepting services from state and federally funded health care entities (Fisher, 2002; Sherman, 2014). Most Anabaptist community members are self-insured, and mutual 
aid is practiced within communities (Gingrich \& Lightman, 2006; Kraybill \& Hurd, 2006). Medical decisions are typically made with input from one's family and community, with seeking the will of God and the collective good of the community being the driving forces in decisionmaking (Garrett-Wright et al., 2016). According to Kotva (2002), "the most fundamental notion of health concerns well-being in our relationship with God and others, especially fellow believers in the church" (p. 3).

Anabaptist groups are also known for their interest in health promotion and the use of natural products. In a study by Gesink and colleagues (2017), a strong interest in basic health tenets such as obtaining adequate sleep and maintaining a good diet were reported. Other authors have noted the predisposition of Anabaptists to use herbal products and supplements to prevent and treat illness instead of seeking formal health care services (Gingrich \& Lightman, 2006; Sharpnack et al., 2010). Anabaptists seek to maintain their health so they can continue to work and be active in their community (Armer \& Radina, 2006; Fisher, 2002).

In addition to valuing spiritual and communal connections, Anabaptists appreciate a sense of connectedness and consistent relationships with their medical providers (Garrett-Wright et al., 2016). Donnermeyer and Friedrich (2006) noted Anabaptists' preference for seeing a medical provider with an understanding of their community and culture. Anabaptist patients have a desire to see an HCP who demonstrates a caring and engaged demeanor and respects the norms of their culture (Garrett-Wright et al., 2016).

Understanding the complexities of Anabaptist health care beliefs can be challenging for HCPs. Anabaptists are a unique population and differ from the typical rural population that some HCPs work with routinely (Rohr et al., 2019). Little research has been conducted on HCPs' understanding of Anabaptist health care beliefs, practices, and preferences. Culturally competent health care for Anabaptist populations can be developed following self-examination of one's own biases, identification of knowledge deficits regarding the population, and experience in delivering care in the population. This study allowed HCPs to report their understanding of Anabaptist health care beliefs, practices, and preferences and to explore lessons learned while care for Anabaptist patients.

\section{Methods}

\section{Participants and Inclusion Criteria}

Sampling for the study included purposive and snowball sampling techniques. Participants for the study were HCPs recruited from the investigators' knowledge of known providers for Anabaptist communities in the southeastern U.S. In addition, some participants were contacted based on recommendations from other participants and Anabaptist community members. Inclusion criteria included being a provider who served Anabaptist communities, age over 18 years, and ability to read and write in English. The study was approved by the Human Subjects Review Board at a state university in the Southeast before data collection began. HCPs participating in the study completed and signed an informed consent document. 


\section{Instrument}

Data was collected using semi-structured interview questions that were developed by the investigators, a group of three researchers who had experience working with members of an Anabaptist community (Appendix). The participants also completed a basic demographic survey that included questions about age, gender, ethnicity, occupation, and locale of practice. Interview questions focused on the HCPs' experiences when providing health care to Anabaptist populations, including the types of issues/health concerns they had addressed with that population and any barriers to care they noted in the population. Participants were asked to discuss ethical, legal, and economic issues that were encountered in care situations with members of Anabaptist communities. There were also questions that asked participants to reflect on the lessons learned from caring for members of an Anabaptist population.

\section{Data Collection Procedures}

Participants were selected in a purposive manner based on the researchers' knowledge of HCPs who provided care in Anabaptist communities in two southern states and one HCP from the Northeast. HCPs were approached in person or by a phone call from one of the investigators and given an explanation of the research project. Those interested in participating in the study signed an informed consent document that included a separate prompt to initial for inclusion of their videotaped responses in a continuing education program. HCPs who agreed to participate were interviewed in a location that was convenient for them (e.g., their office or home) based on their preference, and a videographer recorded their interviews.

\section{Thematic Analyses}

Videotaped interviews of the HCPs' responses to interview questions were analyzed using thematic analysis. Braun and Clark (2006) have reported that thematic analysis provides a systematic technique to identify, analyze, and report themes that appeared in a qualitative data set. The videotaped interviews were transcribed into Microsoft Office Word documents to assist with analysis of content. The initial step in analysis was for each researcher to read through individual interview transcripts several times to make notes on content and areas where common ideas were expressed. Initial codes were developed by each researcher independently, and these codes were subsequently collated into larger themes. After initial codes and themes were developed independently, the researchers met together to review codes and themes. During this time, the researchers worked on defining and clarifying the meaning of themes and determining names for the themes. The transcripts were then reviewed an additional time by each researcher independently, using the themes already identified. The purpose of this final review was to determine the adequacy of the themes in describing the overall data provided by the participants and to select exemplar quotes to be used in dissemination of the research and in development of continuing education materials for HCPs. 


\section{Results}

\section{Demographics}

Eighteen HCPs agreed to participate in the study and completed the videotaped interviews. The participants practiced in three states, with 17 of the 18 participants practicing in the southeastern U.S. The sample was predominately female $(n=11,61.1 \%)$ and all participants were Caucasian $(n=18 ; 100 \%)$. Participant age ranged from 33 to 89 years. Health care professions represented in the sample included physicians $(n=6)$, nurse practitioners $(n=4)$, registered nurses

$(n=5)$, dentists $(n=1)$, and dental hygienists $(n=1)$. There was also one ancillary health care provider who served as a financial counselor $(n=1)$.

\section{Themes}

Thematic analysis revealed seven commonly expressed themes from the larger data set. The themes included the following: 1) Anabaptist communities place value on negotiating their prescribed plan of care, 2) community members take greater responsibility for their health outcomes as compared to mainstream communities, 3) there is increased value placed on direct communication, active listening, and presence in the relationship between the patient and $\mathrm{HCP}, 4)$ there is a tendency to delay care until later in the course of illness, 5) community members are cautious in using mainstream treatments and desire to use natural products when possible, 6) there is a general aversion to legal action, and 7) community members express appreciation for health care that is provided to them. These themes provide insight into HCPs' understanding of what is valued by Anabaptists in health care scenarios and can be compared to what community members report as being of value in interactions with HCPs.

\section{Theme \#1: Value of negotiating plan of care}

Study participants reported that their Anabaptist patients and families had a desire to be active participants in negotiating their care. There was an interest in discussing and seeking an understanding of care options. Negotiations were done in the spirit of cooperation and in a way that promoted the ongoing provider-patient relationship. One HCP noted,

I would say that this is probably the most receptive population to education that I have ever worked with. They do not become angry if you point something out, they are willing to listen, they enjoy feedback, they like to give you feedback, and so I think it is a very reciprocal relationship.

Another HCP also noted the importance of reciprocity in the relationship and the value Anabaptists place on providing feedback during the care negotiations, stating, "They were always receptive to plans of care. Care was always discussed and what they deemed appropriate from their plan; it was very much a decision-making process that was bilateral not unilateral, not 
paternalistic." The HCPs expressed that the Anabaptist community did not always follow all recommended actions, but that disagreement was handled in a respectful manner.

\section{Theme \#2: Greater responsibility for health outcomes}

HCPs in the current study reported an increased level of personal responsibility for health outcomes seen in members of Anabaptist communities. All HCPs in the study noted that their Anabaptist patients and families valued information regarding health promotion. One health professional reported,

I think it is very good to see a community become more informed about health ... they take that to heart and they use that knowledge to improve their community so I think it is very rewarding to know that the education piece and the health promotion piece is really put to use in that community.

Another provider noted that a greater emphasis is placed on preventing illness rather than reacting to illness. This provider reported,

I think there is probably more of an emphasis on health promotion in their community. I think they would like to prevent things. I think they would like to understand how they can be healthier, whether that is through using natural remedies and herbs and other natural products so that they can prevent things. They would rather do that than to end up with a serious illness.

The providers in the study reported that the emphasis on personal responsibility for health and wellness through health promotion and illness prevention is one that is not seen as often in English culture.

\section{Theme \#3: Direct communication, active listening, and presence}

HCPs discussed the differences noted in their communication with Anabaptist patients and those from English culture. They indicated that there was an increased value placed on direct communication, active listening, and being fully present when communicating with Anabaptist community members. One HCP noted that it takes more time to develop the relationship in an Anabaptist community and is helpful to spend time learning about the providers' community and life. The HCP stated, "We really did just try to have an open dialogue with the community, and you know would answer questions that they had about our culture and some of the things that we have done." Another HCP said that the level of communication desired in Anabaptist communities requires a time commitment on the part of the provider. This provider noted, "I would really strongly encourage patience, that it is going to take a while to develop this relationship and perhaps longer than it takes for you to develop relationships with your English patients, but that it can 
happen." HCPs in English communities are used to delivering diagnoses and treatment plans and often talk more than the patient during encounters. HCPs in the current study emphasized the need for active listening with Anabaptists. One stated,

I think that is how you earn their respect to begin with, is to listen to them because then they know that you are truly interested in hearing what they have got to say, and that you are really interested in them as an individual.

Data analysis also revealed that HCPs working with Anabaptist community members have come to recognize the high level of intellect found in these communities. HCPs noted that Anabaptist community members often have a better understanding of medical topics than some members of the English community. With this in mind, HCPs in the study recommended that communication be respectful but to not talk down to the patient. One HCP stated, "They are very educated so they really understand a lot more than what we would give them credit for, and I think that is to our detriment to automatically think that they are undereducated because they are not."

\section{Theme \#4: Delay in care}

Delay in care for routine illnesses was observed by most participants in the study. As noted in a previous theme, members of Anabaptist communities often seek to use natural remedies to treat common illnesses and therefore may not present to English health care settings until later in the course of an illness. An example of this was mentioned by a participant who stated, "They are more do-it-your-selfers in general...they have usually done multiple things to themselves before ever asking for advice." Another HCP reported,

The only time that we saw them in the office is when they failed their home remedies, and quite a bit of them were successful. In our culture outside of the Amish community, we tend to run to the doctor for everything, viruses or bacterial infections. They tend to not come in unless it is related to a persistent infection that does not have a response to their home remedies and that is given adequate time for a virus to run its course.

While HCPs were open to Anabaptists trialing home remedies and delaying care for minor illnesses, there were concerns expressed. One HCP stated, "Sometimes I think they delay treatment a little longer than, perhaps, should be. But that is my opinion, but I do not think access to health care is a big issue." The overall analysis of the participants' responses indicated that HCPs expected some self-imposed delays in care for Anabaptists but felt this was their choice to make.

\section{Theme \#5: Caution about mainstream medicine}

HCPs reported that Anabaptist patients and families are cautious about the use of mainstream

medicine interventions and prefer to try alternative treatments before seeking care from a 
mainstream provider. One participant observed, "They are willing to spend money on holistic medicine. They are willing to spend money on herbal medicine. They are willing to spend money on chiropractors ...but they are reluctant to spend money on regular medicine." A dental provider in the study noted that often they reject commonly practiced medical and dental interventions and providers have difficulty getting them to undergo simple dental procedures. The dentist stated,

So, we would do the cleaning and even beyond that, um, because there are some, um, nonscientifically supported articles out there that, you know, say the dangers of fluoride. And, um, we would always, you know, have to really do a lot of educating to get some to do fluoride treatment afterward.

Participants in the study noted that resistance to mainstream medicine is partially a response to misinformation but also ties back to core values. One HCP summed this up by saying members of Anabaptist communities often feel "that God gave us everything we needed in nature if we would just take time to investigate and explore."

\section{Theme \# 6: Aversion to legal action}

Anabaptist communities are known for limited involvement in legal proceedings and for accepting events that happen as part of the plan of God for their lives. These beliefs have become known to providers, one of whom stated, "Members of the Amish and Mennonite communities do not believe in using the legal system to establish blame or blame for economic gain." This aversion to legal action has relieved the anxiety of HCPs who encounter Anabaptists in health care settings. Another HCP noted that "they are very desirable patients because of their realistic expectations ... decreased liability is going to be associated with them." However, HCPs did not feel that the decreased likelihood of being sued when working with Anabaptists changed the plan of care they offered. As one participant stated,

I would say no, but only because the issues where that would have been brought up, [where it] would have entered my mind that they would not take legal action, it was more of an ethical thing for me, and I had already decided in those situations that ethically I am not going to do this. Regardless of whether I would get sued for it or not.

\section{Theme \# 7: Appreciation for care}

The HCPs in the current study voiced that they felt appreciated by the Anabaptist community members they served. This was in contrast to how some HCPs felt they were treated by members of the English community. One HCP stated, "It seems like they are more appreciative of anything you do. A lot of times patients come in with expectations. You owe them something, and you are required to do certain things that are really not part of your job. Anything you do for the Amish and Mennonite community, they seem so appreciative." This sentiment was echoed by another 
participant who said, "They are an extremely grateful community. They are happy for anything that you can do for them and sometimes it is nothing other than listening to them."

\section{Discussion}

The current study provides a qualitative analysis of what HCPs serving Anabaptist communities view as the core health care beliefs and preferences to be aware of when providing care to this population. The findings of this study mirror findings in previous studies on Anabaptist communities and their health care beliefs and preferences. The value of a reciprocal and caring relationship between the provider and the community was noted as being important in this study. This is an accurate assessment of community desires based on the responses of community members reported in previous studies. According to Garrett-Wright et al. (2016), Anabaptist community members expected the provider to be engaged and to display a caring attitude that respected the patient's cultural norms. Rohr et al. (2019) similarly found that Anabaptists prefer to receive patient information directly through conversation with the provider rather than by handouts or phone calls when obtaining care within the English health system. The HCPs in the current study indicated that it takes time to establish and maintain good relationships with Anabaptist community members. Assuring adequate time to discuss conditions and treatment options during health care interactions with this population can promote trust and mutual respect.

The study also demonstrates that HCPs understand that the use of alternative treatment, such as the use of herbal preparations and nutritional supplements, is important to Anabaptists. This finding is similar to the results of the study by Gesink et al. (2017) in which 53\% of participants endorsed seeking care from a complementary health provider and $42 \%$ indicated willingness to seek care from alternative health care providers. The use of herbal products and nutritional supplements reflects their belief in the provision of God to give them what they need to be healthy, as well as their caution about using mainstream medicine. This finding is consistent with those of previous studies that noted Anabaptist community members often sought to limit drug use and rely on natural products to prevent or treat illnesses (Garrett-Wright et al., 2016; Sharpnack et al., 2010). This finding is also congruent with a survey conducted by Rohr et al. (2019) that found only about $36 \%$ of participants sought out doctors as an important source of information on health issues. Given the broader use of herbal products and supplements in Anabaptist populations, it is crucial that providers seek education on common remedies used by Anabaptist groups in their practice region. In addition, a better understanding of the remedies used in these communities can help HCPs educate community members on when seeking formal medical care is necessary, complications that can arise from delaying care, and potential adverse effects from natural remedies.

The finding that Anabaptist populations report avoiding legal action in health care has been reported (Talpos, 2016); however, this study provides an understanding of how HCPs view this reality. HCPs in the current study discussed that knowing one will not face legal repercussions when dealing with this population does decrease anxiety in their professional practice. The HCPs 
in the current study voiced that Anabaptists believe God's hand is in the events of life and that is part of the reasoning behind avoiding legal action in health care and other settings. Providers in the current study did report that the standard of care provided was based on their own ethical and professional values and that the reduction in the risk of legal action with this population did not change the standard of care provided.

The findings from the current study offer the HCPs' views of key issues in caring for the Anabaptist population and share many commonalities with findings from previous studies regarding the Anabaptists themselves. Key findings included the value of natural products and delaying care at times, the value of reciprocal relationships between provider and patient, and differences noted in taking legal action and showing appreciation for care provided. The findings cannot be transferred to all segments of the U.S. population due to the qualitative nature of the data collected and the limited geographic area where the HCPs in the study practiced. However, findings from this study support the need for HCPs to have education on the health care values and beliefs of this unique population so that respectful care can be provided. Information gained from the current study was used to develop a continuing education program for HCPs regarding care for Anabaptists, which is available nationally on a training website.

\section{Strengths and Limitations}

There are strengths and limitations associated with the current study. The qualitative design allowed for HCPs to provide in-depth data regarding experiences with Anabaptist populations and the lessons learned in these exchanges. Transferability of the data was enhanced by continuing to collect data until saturation was obtained. The investigators also had an audit trail consisting of notes and e-mails reflecting the process of how codes were identified and collapsed into larger themes, as well as how themes were defined and confirmed. These steps helped to ensure the dependability of the data. Credibility is enhanced by the prolonged engagement of the investigators with the topic of health care in the Anabaptist population. The investigators have worked from 10 to 25 years with Anabaptist populations. Sampling in the study can be considered a limitation. The sample is drawn primarily from HCPs working in southeastern states and may not represent the experience of all HCPs who care for Anabaptists.

Future research should be conducted in other parts of the United States and other countries to determine if the experience of HCPs working with Anabaptist populations is congruent with findings from the current study. In addition, it would be important to examine the perceptions of a more varied set of HCPs. The current study's sample consisted of mostly physicians and nurses, while other health disciplines were not represented. Future research should include providers such as physical therapists, dieticians, and social workers.

\section{Conclusion}

HCPs in the current study viewed Anabaptists as appreciative of the care they received and less willing to take legal action if medical errors occurred. They also viewed Anabaptists as desiring a 
closer and more reciprocal relationship with their HCP. The desire to use natural remedies and delaying care are also consistently mentioned by HCPs who participated in the study. In many instances, the findings from interviewing HCPs about their patients' health care beliefs and preferences are supported by community members themselves (Garrett-Wright et al., 2016). The results expand the literature on how HCPs work with Anabaptist populations and the views that guide their practice when caring for this population. Results indicated a positive view of Anabaptist culture and a good working knowledge of issues that are important to the population. The findings indicate that HCPs in the study did practice with sensitivity in the Anabaptist culture.

\section{References}

Armer, J. M., \& Radina, M. E. (2006). Definition of health and health promoting behaviors among Midwestern Old Order Amish families. The Journal of Multicultural Nursing \& Health, 12(3), 44-53.

Braun, V., \& Clarke, V. (2006). Using thematic analysis in psychology. Qualitative Research in Psychology, 3(2), 77-101. https://doi.org/10.1191/1478088706qp063oa

Donnermeyer, J. E., \& Friedrich, L. (2002). Amish society: An overview reconsidered. Journal of Multicultural Nursing \& Health, 8(3), 6-14. (Published again in 2006: Journal of Multicultural Nursing \& Health, 12(3), 35-43.)

Edwards, M. (2013). Health literacy, health disparities and culturally responsive primary care. In T. Buttaro, J. Tyrbulski, P. Bailey, \& J. Sandberg-Cook (Eds.), Primary care: A collaborative practice (4th ed., pp. 34-39).

Fisher, K. (2002). Lessons learned while conducting research within an Amish community. Journal of Multicultural Nursing \& Health, 8(3), 21-28.

Flynn, P., Betancourt, H., Emerson, N., Nunez, E., \& Nance, C. (2020). Health professional cultural competence reduces the psychological and behavioral impact of negative healthcare encounters. Cultural Diversity and Ethnic Minority Psychology, 26(3), 271-79. https://doi.org/10.1037/cdp0000295

Garrett-Wright, D., Main, M. E., \& Jones, M. S. (2016). Anabaptist community members' perceptions and preferences related to healthcare. Journal of Amish and Plain Anabaptist Studies, 4(2), 187-200. https://doi.org/10.18061/1811/79932

Gesink, D., Leach, J., McBride, K., \& Bergin-Payette, K. (2017). Health priorities and healthseeking behaviors of Old Order Anabaptists in Southern Ontario with emphasis on cancer screening. Journal of Transcultural Nursing, 28(6), 566-72. https://doi.org/10.1177/1043659616666325

Gingrich, L., \& Lightman, E. (2006). Striving toward self-sufficiency: A qualitative study of mutual aid in an Old Order Mennonite community. Family Relations, 55(2), 175-89. https://doi.org/10.1111/j.1741-3729.2006.00368.x 
Kotva, J., Jr. (2002). The Anabaptist tradition: Religious beliefs and healthcare decisions. Park Ridge Center for the Study of Health, Faith, and Ethics. https://www.advocatehealth.com/assets/documents/faith/anabaptist_final.pdf

Kraybill, D. B., \& Hurd, J. P. (2006). Horse-and-buggy Mennonites: Hoofbeats of humility in a postmodern world. University Park, PA: Pennsylvania State University Press.

Lancaster Mennonite Historical Society. (2019, May 27). Broad census counts more Anabaptists. Mennonite World Review. http://mennoworld.org/2019/05/27/news/broad-census-countsmore-anabaptists/

Rohr, J., Spears, K., Geske, J., Khandalavala, B., \& Lacey, M. (2019). Utilization of health care resources by the Amish of a rural county in Nebraska. Journal of Community Health, 44(6), 1090-97. https://doi.org/10.1007/s10900-019-00696-9

Sharpnack, P., Griffin, M., Benders, A., \& Fitzpatrick, J. (2010). Spiritual and alternative healthcare practices of the Amish. Holistic Nursing Practice, 24(2), 64-72. https://doi.org/10.1097/HNP.0b013e3181d39ade

Sherman, C. (2014). Respecting cultural differences: Alternatives for the Amish community to combat health care costs. Undergraduate Research Journal for the Human Sciences. http://www.kon.org/urc/v13/sherman.html

Spector, R. (2017). Cultural Diversity in Health and Illness. New York, New York. Pearson. Talpos. S. (2016). The Amish understand a crucial thing about modern medicine that most Americans don't. Quartz. https://qz.com/695101/the-amish-understand-a-crucial-element-ofmodern-medicine-that-most-americans-dont/

Unver, V., Uslu, Y, Kocatepe, V., \& Kuguoglu, S. (2019). Evaluation of cultural sensitivity in healthcare service among nursing students. European Journal of Educational Research, 8(1), 257-65. https://doi.org/10.12973/eu-jer.8.1.257

\section{Appendix: Health Care Provider Interview Questions}

Demographics

- Gender

- Age

- Occupation (type profession)

- Location of practice

Questions for Health Care Providers

1. Tell me about your experience in providing health care to members of Amish/Mennonite communities?

2. In your opinion, what would you consider to be the major health issue/concern of Amish/Mennonite individuals? Of Amish/Mennonite communities?

3. What do you think is the major barrier to health care for the Amish/Mennonite populations? 
4. Describe differences/similarities in providing health care to members of Amish/Mennonite populations when compared to mainstream society (English people).

5. What are some challenges you have encountered when providing health care to members of Amish/Mennonite communities?

6. What are some of the major rewards received from serving this diverse population?

7. Have you encountered any ethical issues when working with Amish/Mennonite populations? If so, please describe.

8. Amish/Mennonite populations are known for the use of alternative medicine. Describe your thoughts about this practice in today's modern medical practice.

9. What influence do you think economics plays in the health care of Amish/Mennonite populations?

10. Do you think the family structure in the Amish/Mennonite families influence health care? If so, explain.

11. During your tenure working with Amish/Mennonite populations, share any lessons you have learned about maintaining cultural sensitively.

12. What do you consider to be the most important action health care providers can make to enhance the relationship between members of Amish/Mennonite communities and health care providers?

13. Share any language or communication challenges you have experienced when working with members of the Amish/Mennonite communities.

14. Members of Amish/Mennonite communities do not believe in using the legal system to establish blame or for economic gain. Share your thoughts about how this belief influenced the health care you provided for this population. 